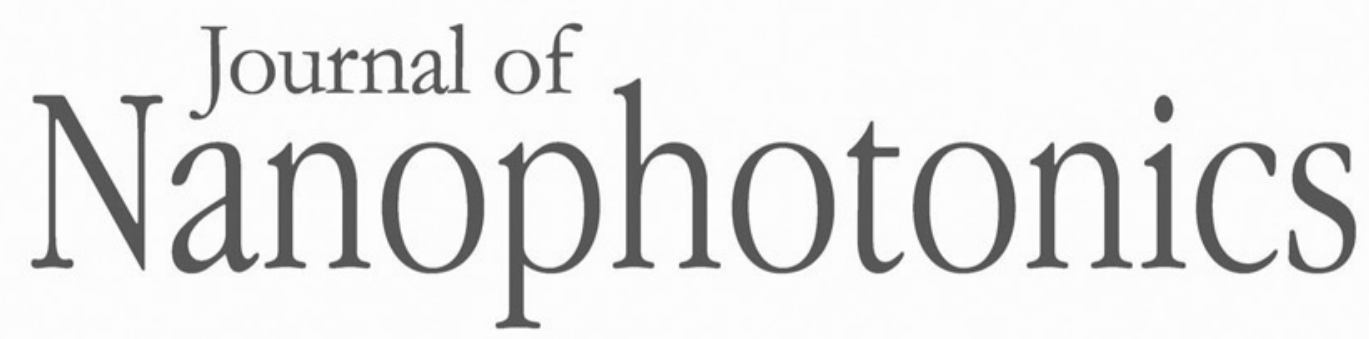

\title{
Publisher's note: Broadband absorption enhancement in organic solar cells using refractory plasmonic ceramics
}

\author{
Sara Magdi \\ Dengxin Ji \\ Qiaoqiang Gan \\ Mohamed A. Swillam
}




\title{
Publisher's note: Broadband absorption enhancement in organic solar cells using refractory plasmonic ceramics
}

\author{
Sara Magdi, ${ }^{a}$ Dengxin Ji, ${ }^{b}$ Qiaoqiang Gan, ${ }^{b}$ and Mohamed A. Swillam ${ }^{\text {a,c }}$ \\ ${ }^{\mathrm{a}}$ American University in Cairo (AUC), Nanotechnology Program, \\ AUC Avenue New Cairo, Cairo, Egypt \\ ${ }^{b}$ University at Buffalo, The State University of New York, \\ Department of Electrical Engineering, Buffalo, New York, United States \\ ${ }^{\mathrm{c}}$ American University in Cairo, Department of Physics, AUC Avenue New Cairo, Cairo, Egypt
}

This article [J. Nanophoton. 11(4), 046001 (Jan 6, 2017)] originally was published with an incorrect citation identifier (CID). It was republished with a corrected CID on 11 January 2017. The updated citation is shown below:

S. Magdi et al., "Broadband absorption enhancement in organic solar cells using refractory plasmonic ceramics," J. Nanophoton. 11(1), 016001 (2017).

() 2017 Society of Photo-Optical Instrumentation Engineers (SPIE) 